\title{
Design of Hydraulic Bushing and Vehicle Testing for Reducing the Judder Vibration
}

\author{
Youngman $\mathrm{Kim}^{1}$, Sangjin Jeong ${ }^{1}$, Van-Quyet Nguyen ${ }^{1}$, Kwangsuck Boo ${ }^{2}$ and Heungseob Kim²,a \\ ${ }^{1}$ Graduate school of Mechanical Engineering, Inje University, 50834 Gimhae, Gyoungnam, Korea \\ ${ }^{2}$ HSV-TRC Center, Inje University, 50834, Gimhae, Gyoungnam, Korea
}

\begin{abstract}
Generally, judder vibration is a low-frequency vibration phenomenon caused by a braking force imbalance that occurs when a vehicle is lightly decelerated within a range of 0.1 to $0.2 \mathrm{~g}$ at a speed of 120 to $60 \mathrm{~km} / \mathrm{h}$. This comes from the change in the brake disk thickness (DTV), which is mainly caused by the side run-out (SRO) and thermal deformation. The adoption of hydro-bushing in the low arm $\mathrm{G}$ bushings of the vehicle front suspension has been done in order to provide great damping in a particular frequency range $(<20 \mathrm{~Hz})$ in order to prevent this judder vibration from being transmitted to the body. The hydro bushing was formulated using a lumped parameter model. The fluid passage between the two chambers was modelled as a nonlinear element such as an orifice, and its important parameters (resistance, compliance) were measured using a simplified experimental setup. The main design parameters are the ratio of the cross-sectional area of the chamber to the fluid passage, the length of the fluid passage, etc., and their optimal design is such that the loss angle is greater than $45^{\circ}$ in the target frequency range of 10 to $20 \mathrm{~Hz}$. The hydro bushing designed for reducing the judder vibration was prepared for the actual vehicle application test and applied to the actual vehicle test. In this study, the proposed hydro bushing was applied to the G bushing of the low arm of the front suspension system of the vehicle. The loss angle of the manufactured hydro bushing was measured using acceleration signals before and after passing through the bushing. The actual vehicle test was performed on the noise dynamometer for the performance analysis of the judder vibration reduction.
\end{abstract}

\section{Introduction}

Generally, in vibration theory, it is known that high stiffness and large damping are easy to cut off low frequency and large displacement vibration, low stiffness and small damping are advantageous for high frequency and small displacement vibration. The conventional rubber bushing meets the contradictory requirements of sufficient attenuation for large displacement vibrations in the rigid body mode below $100 \mathrm{~Hz}$ and high decoupling of the structural noise in the high frequency range. The requirements of this contradictory bushing design have required the development of hydrobushing which can enhance the damping force through the fluid and control the stiffness and damping in the frequency range of interest. The principle of fluid damping technology is already known in engine mount technology, but there are constraints in design and material selection for application in chassis

\footnotetext{
${ }^{\mathrm{a}}$ Corresponding author : mechhsk@inje.ac.kr
} 
components. Nevertheless, the suspension application of the hydro-bushing was applied to the lower arm $\mathrm{G}$ bushing of the front suspension in Fig. 1 to reduce the steering wheel judder vibration due to the braking force imbalance. [1 2]
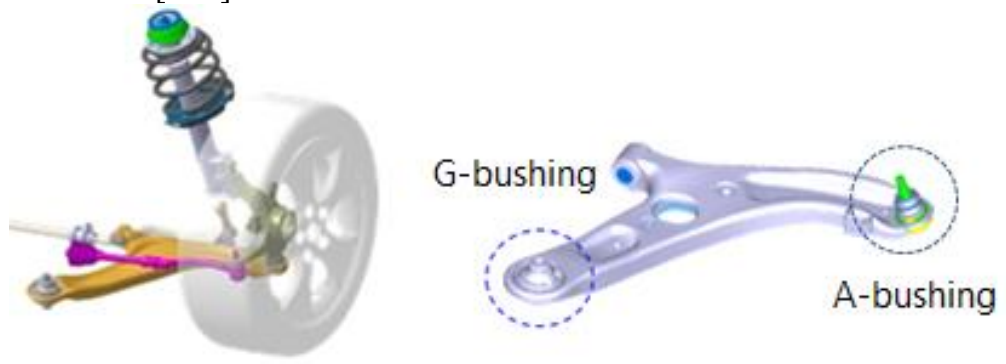

Figure 1. G bushing position of lower arm in the MacPherson suspension

Judder vibration is a low-frequency vibration phenomenon in the range of $10 \sim 20 \mathrm{~Hz}$ caused by unbalance of braking force when the vehicle is braking at a light deceleration of $0.1 \sim 0.2 \mathrm{~g}$ at a speed of $120 \mathrm{~km} / \mathrm{h}$, which is a highway driving speed. Judder is known to be caused by disc thickness variation (DTV), which is caused by side run-out (SRO) during manufacturing and thermal deformation resulting from the braking process. Compared to conventional rubber bushings, hydro bushings can have high viscous damping in certain frequency ranges, but have amplitude and frequency dependencies. The design core of the hydro bushing is to determine the peak frequency and magnitude of the loss angle. The main design parameters are rubber spring constant, fluid characteristics, and the shape of the chamber and inertia track.

In this study, the frequency characteristics of the hydro bushing applied to the G bushing of the front suspension low arm were designed to effectively reduce the judder vibration. Also, the performance of the hydro bushing was verified by performing the actual vehicle test on the noise dynamo using the vehicle with the hydro bushing manufactured.

\section{Characteristic design of hydrobushing}

The lumped parameter model (LP model) as shown in Fig.2 is widely used to predict the characteristic analysis of the hydrobushing [3 4]. The hydro bushing consists of two chambers connected by fluid passages, rubber elements and a metal sleeve on the inside and on the opposite side. The rubber elements consist of a Kelvin-Voigt model with frequency-invariant stiffness $K_{R}$ and viscous damping coefficient $C_{R}$. The two chambers are defined by compliance $C_{1}, C_{2}$ controlled by the rubber portion and the fluid portion including the air. The fluid passage is modeled by a resistance element $R_{i}$ and an inertance element $I_{i}$.

When dynamic displacement is applied to the inner sleeve of the hydrobushing, the dynamic pressure of both chambers is

$$
\begin{aligned}
& C_{1} \dot{p}_{1}(t)=-A_{1} \dot{x}(t)-q_{i}(t) \\
& C_{2} \dot{p}_{2}(t)=A_{2} \dot{x}(t)+q_{i}(t)
\end{aligned}
$$

Where $A_{1}, A_{2}$ represents the effective pumping area of each chamber, and $q_{i}$ represents the volumetric flow rate of the fluid flowing through the fluid passages connecting the chambers. The conservation of momentum between the chamber and the fluid passage can be expressed as Equation (3).

$$
p_{1}(t)-p_{2}(t)=I_{i} \dot{q}_{i}(t)+R_{i} q_{i}(t)
$$

The forces transmitted by the two paths to the outer sleeve are as follows.

$$
f_{T}(t)=\left[C_{R} \dot{x}(t)+K_{R} x(t)\right]+\left[A_{2} p_{2}(t)-A_{1} p_{1}(t)\right]
$$




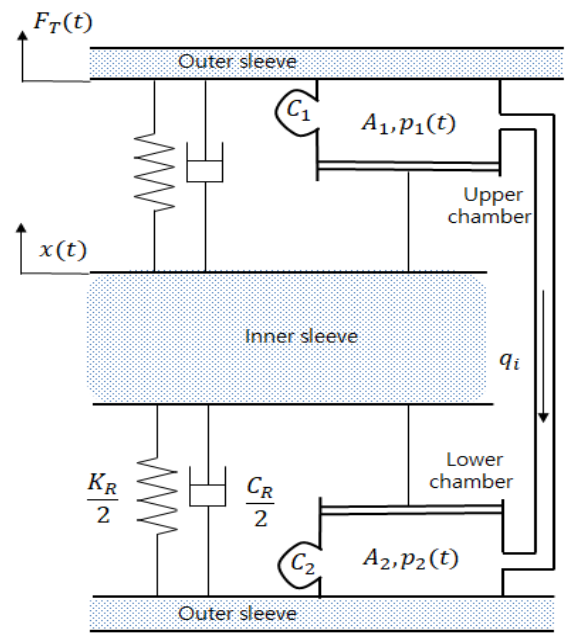

Figure 2. Lumped parameter model of hydrobushing

The resistance $R_{i}\left(q_{i}\right)$ of the fluid passage can be modeled nonlinearly using the same orifice model as Eq. (5) [5] .

$$
R_{i}\left(q_{i}\right)=\frac{\Delta p}{q_{i}}=\lambda \frac{\rho}{2 A_{i} C_{d}^{2}}\left|q_{i}(t)\right|=c_{R}\left|q_{i}(t)\right|
$$

Where $C_{d}$ is the expansion coefficient, $A_{i}$ is the cross-sectional area of the fluid passage, and $c_{R}$ is the resistance coefficient. $\lambda$ is a coefficient that adjusts the difference between experimental and theoretical models. The hydro bushing with nonlinear characteristics for sinusoidal input is tried to obtain the transfer function for the dynamic stiffness and loss angle. If the fluid channel is made nonlinear as shown in Eq. (5), Eq. (3) becomes as shown in Eq. (6). If the two chambers have the same cross-sectional area, Eq. (7) can be obtained by combining Eq. (1) and Eq. (2).

$$
\begin{gathered}
p_{1}(t)-p_{2}(t)=I_{i} \dot{q}_{i}(t)+c_{R}\left|q_{i}(t)\right| q_{i}(t) \\
I_{i} \ddot{q}_{i}(t)+2 c_{R} q_{i}(t) \dot{q}_{i}(t) \operatorname{sgn}\left[q_{i}(t)\right]+k q_{i}(t)=\alpha_{x} \dot{x}(t)
\end{gathered}
$$

Where $\mathrm{k}=1 / C_{1}+C_{2}, \alpha_{x}=-\left[A_{1} / C_{1}+A_{2} / C_{2}\right]$. Assuming that the input displacement of the hydrobushing inner sleeve is $x(t)=X \sin (\omega t-\varphi)$, and the flow rate of the resulting hydraulic passage is $q(t)=Q_{1} \sin (\omega t)$, then Eq. (7) is

$$
\begin{gathered}
k Q_{1} \sin (\omega t)+c_{R} Q_{1}^{2} \omega \sin (2 \omega t) \operatorname{sgn}[\sin (\omega t)]-I \omega^{2} Q_{1} \sin (\omega t) \\
=\alpha_{x} X \omega[\cos (\omega t) \cos \varphi+\sin (\omega t) \sin \varphi]
\end{gathered}
$$

Eq. (8) can be simplified by using the Fourier series for $\sin (2 \omega t) \operatorname{sgn}[\sin (2 \omega t)]$, as shown in Eq. (9).

$$
\begin{gathered}
\varphi=\tan ^{-1} \frac{\sqrt{2}\left(k-I \omega^{2}\right)}{\sqrt{-\left(k-I \omega^{2}\right)^{2}+\sqrt{\left(k-I \omega^{2}\right)^{4}-4\left(\frac{8 \alpha_{x} X c_{R} \omega^{2}}{3 \pi}\right)^{2}}}} \\
Q_{1}^{2}=\frac{-\left(k-I \omega^{2}\right)^{2}+\sqrt{\left(k-I \omega^{2}\right)^{4}-4\left(\frac{8 \alpha_{x} X c_{R} \omega^{2}}{3 \pi}\right)^{2}}}{\frac{2\left(8 c_{R} \omega\right)^{2}}{(3 \pi)^{2}}}
\end{gathered}
$$

The flow rates in Eq. (9) and Eq. (10) are as follows.

$$
Q(\omega)=Q_{1} \cos \varphi+i Q_{1} \sin \varphi
$$


And the dynamic stiffness and loss angle can be obtained by Eq. (4).

$$
K_{d}(\omega)=K_{R}+i \omega C_{R}+\gamma+\left(\frac{A_{1}}{C_{1}}+\frac{A_{2}}{C_{2}}\right) \frac{Q(\omega)}{i X \omega}
$$

In Eq. (12), the magnitude and phase of $K_{d}(\omega)$ correspond to the tangent and loss angles, respectively.

\section{Simulation for predicting the performance characteristics}

A simulation for predicting the dynamic stiffness and loss angle of hydro bushings with nonlinear elements such as fluid passages was performed. The developed hydro bushing is designed to reduce judder vibration caused by instability caused by friction during braking process. It is designed to have a loss angle of $30^{\circ}$ or more in the frequency range of $10 \sim 20 \mathrm{~Hz}$.

\subsection{Design parameters}

The developed hydro bushing is shown in Fig. 3. The damping force generated during the flow of the fluid due to the long fluid passage is utilized. Table 1 shows the main design parameters of the hydrobushing. The design is designed to maximize the loss angle in the frequency range of the judder oscillation through the optimal combination of fluid passages and chambers.

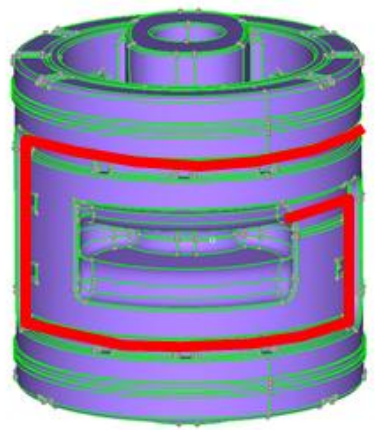

Figure 3. Developed hydrobushing with long flow passage

Table 1. Geometric and material properties

\begin{tabular}{ccc}
\hline Part & Parameter & Value \\
\hline \multirow{2}{*}{ Rubber } & Spring constant, $[\mathrm{N} / \mathrm{mm}]$ & 430 \\
\cline { 2 - 3 } & Damping coefficient, $[\mathrm{Ns} / \mathrm{mm}]$ & 0.1 \\
\hline \multirow{2}{*}{ Inertia track } & Length, $[\mathrm{mm}]$ & 532.1 \\
\cline { 2 - 3 } & Cross sectional area, $\left[\mathrm{mm}^{2}\right]$ & 8.4 \\
\hline Chamber & Pumping area, $\left[\mathrm{mm}^{2}\right]$ & 5609 \\
\hline \multirow{2}{*}{ Fluid } & Viscosity, $\left[\mathrm{Ns} / \mathrm{mm}^{2}\right]$ & $2.5 \mathrm{e}-8$ \\
\cline { 2 - 3 } & Density, $\left[\mathrm{Ns}^{2} / \mathrm{mm}^{4}\right]$ & $1.1 \mathrm{e}-9$ \\
\hline
\end{tabular}

\subsection{Frequency characteristics}

The characteristics of the hydrobushing were obtained by increasing the sinusoidal amplitude of $1 \mathrm{~mm}$ to $5 \sim 50 \mathrm{~Hz}$ at intervals of $1 \mathrm{~Hz}$ and measuring the dynamic stiffness and loss angle at each frequency. The results are compared with those predicted by Eq. (12), and the comparison results are shown in Fig. 4. In Fig. 4(a), the predicted results for the dynamic stiffness differ from the experimental values in the region above $15 \mathrm{~Hz}$, but the prediction of the loss angle in Fig. 4(b) accurately predicts the 
position and the value of the maximum loss angle. Therefore, it was confirmed that the algorithm for predicting the hydro-bushing dynamic characteristics was accurately created.

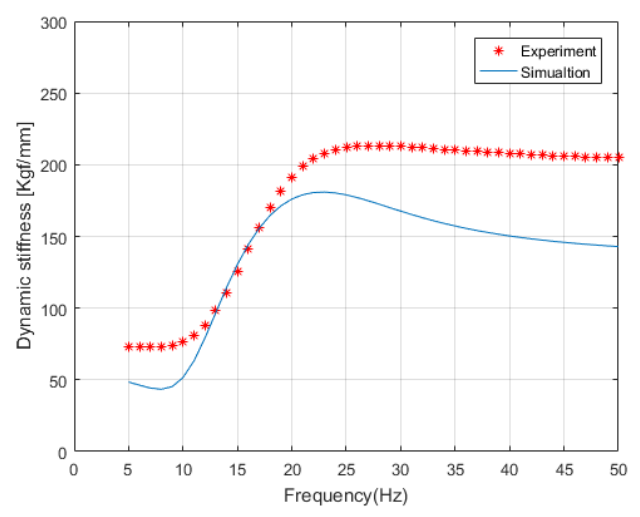

a) Dynamic stiffness

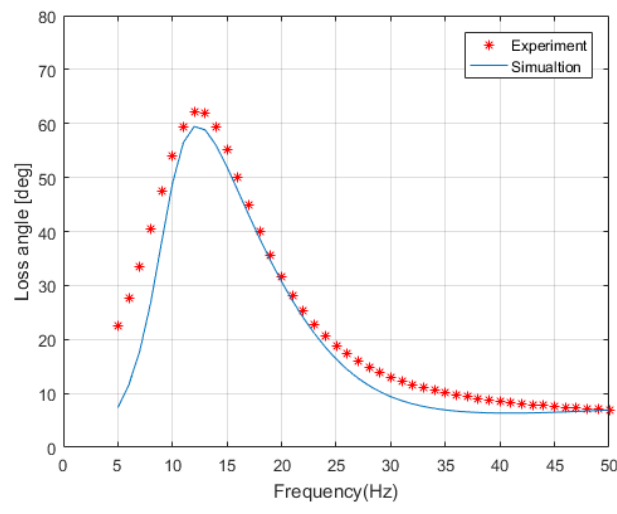

b) Loss angle

Figure 4. Comparisons between experimental and predicted results of the dynamic characteristics

\section{Conclusions}

In order to predict the accurate dynamic performance of the hydrobushing modeled by the lumped parametric model, this study conducted a measurement experiment on the resistance of the fluid passages and chamber compliance with nonlinear characteristics and reflected them in the prediction algorithm. It is found that the predicted results for the dynamic stiffness and loss angles can predict the peak frequency and magnitude accurately with experimental results. Especially, it was confirmed that a large loss angle value was obtained in a desired frequency range so as to reduce the judder vibration.

\section{ACKNOWLEDGEMENT}

This research was supported by the Ministry of Trade, Industry \& Energy (MOTIE), Korea Institute for Advancement of Technology (KIAT) through the Encouragement Program for The Industries of Economic Cooperation Region. (R0004519). This research was supported by The Leading Human Resource Training Program of Regional Neo industry through the National Research Foundation of Korea (NRF) funded by the Ministry of Science, ICT and future Planning. (2016H1D5A1910490)

\section{References}

1. W. Sauer, Y. Guy, SAE 2003-01-1475, (2003)

2. L. Min, T. Yougjun, and W. Enjung, SAE 2010-01-0506, (2010)

3. T. Chai, J. T. Dreyer, and R. Singh, Mechanical Systems and Signal Processing, 56, 92 (2015)

4. W. B. Shangguan, C. Xu, Journal of Vibration and Shock, 26, 7 (2007)

5. T. Chai, R. Singh, and J. Dreyer, SAE 2013-01-1924 (2013) 